\title{
Hyaline articular cartilage dissected by papain: light and scanning electron microscopy and micromechanical studies
}

\author{
PATRICIA O'CONNOR, JANET D. BRERETON, AND D. L. GARDNER
}

From the Department of Histopathology, University of Manchester, Oxford Road, Manchester M13 9PT, and the University Hospital of South Manchester, Withington, Manchester M20 8LR

SUMMARY Papain was used to digest the hyaline femoral condylar cartilages of 30 adult Wistar rats. Matrix proteoglycan degradation was assessed by the light microscopy of paraffin sections stained with toluidine blue. The extent of surface structural change was estimated by scanning electron microscopy, and the structural integrity of the hyaline cartilage tested by the controlled impact of a sharp pin. The results demonstrated an early loss of cartilage metachromasia, increasing with time of papain digestion, and an alteration in the shape of the cartilage splits produced mechanically. Accompanying the loss of matrix material was a conspicuous sequence of morphological changes in cartilage surface structure. The results confirm a close relationship between matrix integrity, mechanical properties, and fine structure.

An understanding of microscopic structure is essential for the interpretation of the mechanical behaviour of hyaline articular cartilage, of joint lubrication, and of the mode of cartilage disruption and failure in osteoarthrosis. Although cartilage morphology has been exhaustively studied by light microscopy (LM) ${ }^{1-4}$ and electron microscopy (EM), ${ }^{5-11}$ and exposure of the internal structure for analysis has been achieved by cutting, fracturing, freezefracturing, and sectioning-techniques that incur displacement and deformation of the tissue components-there is little direct evidence to show that enzymatic degradation prejudices mechanical stability. The purpose of the present experiment was to use an enzyme to remove hyaline cartilage from the articulating surface to expose successive layers of tissue for scanning electron microscopic (SEM) examination. The structural integrity of the digested material could then be tested by the method of pinpricking standardised in a previous study. ${ }^{12}$ The shape and alignment of the splits caused by pricking reflect the collagen fibre arrangement at the cartilage surface. $^{13}$

The thiol proteinase papain was selected. Papain attracted notoriety when the denatured enzyme was

\footnotetext{
Accepted for publication 3 June 1983.

Correspondence to Dr P. O'Connor, Department of Histopathology, University Hospital of South Manchester, Nell Lane, Withington, Manchester M20 8LR.
}

shown to be capable of causing collapse of rabbit ears. ${ }^{14}$ It has been widely employed as a tool to prepare cartilage for chemical analysis. This enzyme has also been used to provoke experimental arthritis ${ }^{15}$ and was selected in the present experiment because of earlier observations ${ }^{16}$ (Inoue $S$, unpublished) that demonstrated its efficacy in degrading articular surfaces before SEM. Papain has also been used previously to degrade blocks of articular cartilage before picro-sirius red polarisation microscopy ${ }^{17}$ and transmission electron microscopy (TEM). ${ }^{1 \times}$

The procedure adopted in this study was to digest whole rat femoral condyles with papain; to examine the response of the degraded tissue to pin-prick testing $^{12}{ }^{13}$; and to study the pricked and non-pricked digested material by SEM. Light microscopy was used to examine full-thickness perpendicular cartilage sections at various stages of digestion and in particular to analyse those early changes in the intercellular matrix that might not be detectable by SEM.

\section{Materials and methods}

Thirty-four 330-350 g inbred Wistar rats of a Manchester strain were killed and the lower ends of both femurs removed. Twenty-six pairs of condyles were used for SEM; 42, including 8 controls, for LM. A further 38 normal, non-digested femoral condyles 
were available from a previous study ${ }^{13}$ for comparison; of these 22 had been pin-pricked.

Enzyme digestion. Condyles were kept moist with isotonic saline before suspension in a solution of papain (EC No. 3. 4. 22. 2 Sigma) MW 20700 , obtained in crystalline form from papaya latex. The enzyme was made up in a buffer solution containing $\mathrm{Na}_{2} \mathrm{PO}_{4}(0.05 \mathrm{M})$ and EDTA $(0.01 \mathrm{M})$. L-cysteine hydrochloride $(0.05 \mathrm{M})$ was also added to the buffer to reduce the papain to its active form. The $\mathrm{pH}$ was then adjusted to 6.5 with $\mathrm{NaOH}$. Specimens were digested in an enzyme solution (17 units $/ \mathrm{ml})$ at $37^{\circ} \mathrm{C}$ with regular agitation. Digestion times were 15-240 min for specimens used for SEM, and 2-120 min for condyles processed for LM. After digestion half of each group of specimens were subjected to standard pin-prick testing. ${ }^{12}$ Another 8 specimens were incubated for 2-30 min with enzyme that had been inactivated by boiling. In all cases digestion was terminated by plunging the tissue into chilled isotonic saline.

Pin-prick technique. Each specimen was secured to the pin-prick apparatus by a fine rod inserted into the residual femoral shaft of the inverted condyle. The rod was positioned above a ball-and-socket joint situated on an adjustable platform. This arrangement enabled a series of pricks to be made over the entire articulating surface. The cartilage was pricked with a $0.5 \mathrm{~mm}$ diameter pin attached to the underside of a pivoted arm. The position of weights on this arm and the height of release of the pin above the articular surface were kept constant and determined the applied force and the depth of penetration.

Light microscopy. Tissue was fixed in neutral buffered formalin, decalcified in ethylenediamine tetra-acetic acid (EDTA), and prepared as 4-6 $\mu \mathrm{m}$ paraffin sections, which were stained with haematoxylin and eosin (HE) and with alcoholic toluidine blue (TB).${ }^{19}$ Sections from regions 1,3 , and 4 (Fig. 1 ) were systematically analysed.

Scanning electron microscopy. Cartilage blocks were collected into cold $\left(4^{\circ} \mathrm{C}\right)$ cacodylate buffered glutaraldehyde, $\mathrm{pH} 7 \cdot 4$, prepared for SEM by an optimum technique ${ }^{20}$ and sputter-coated with goldpalladium. Each specimen, divided into 4 anatomical regions for assessment ${ }^{12}$ (Fig. 1), was examined in a Cambridge S4 10 SEM at an accelerating voltage of $20 \mathrm{kV}$.

\section{Results}

\section{CONTROLS}

Normal nondigested cartilage. The condylar cartilage was 6-14 cells thick; the uncalcified tissue was 4-7 cells in depth with a 2-4 cell layer of flattened cells at the surface, zone I. The articulating surface formed an intact layer with fibrous tissue overlying hyaline

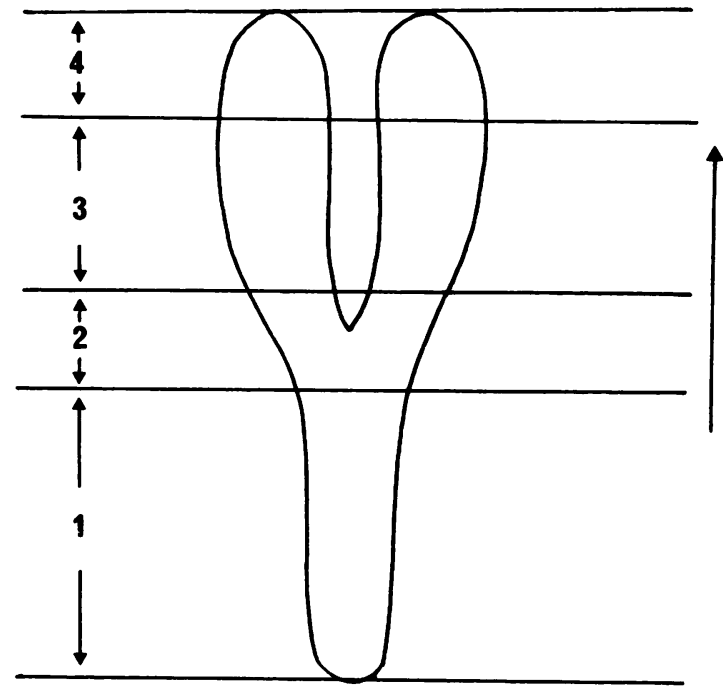

Fig. 1 Mercatorial projection of the articulating surfaces of rat lower end of femur illustrating 4 regional divisions. Arrow indicates longitudinal axis.

cartilage at the periarticular margins. Necrotic cells or empty lacunae were absent. In TB-stained sections there were pale staining regions in the pericellular matrix and in a narrow $\sim 2 \mu \mathrm{m}$ band superficially. On SEM examination the surface was covered by a characteristic array of tertiary (3ry) depressions, 20-30 $\mu \mathrm{m}$ in diameter. Isolated depressions contained central prominences. At magnifications greater than $\times 5000$ the surface was amorphous. The splits caused by pin-pricking penetrated to the tideline, where they frequently propagated along the uncalcified/calcified cartilage interface; they were often deviated by chondrocyte lacunae. Secondary crack formation created irregular split outlines in perpendicular section. En face the splits were elongated, unidirectional, and layered (Fig. 2A).

Normal cartilage incubated with inactivated enzyme. Condyles treated with heat-inactivated enzyme had LM appearances similar to those of normal tissue.

TREATED

Cartilage degraded for 2-15 minutes. There was a loss of TB-staining from a discrete band at the articulating surface after $2 \mathrm{~min}$ digestion (Fig. 3a). This band, thicker in region 1 than in 3 and 4, represented $15-25 \%$ of the uncalcified cartilage after 5 min treatment. Isolated, empty lacunae were now visible superficially. The width of the non-staining 


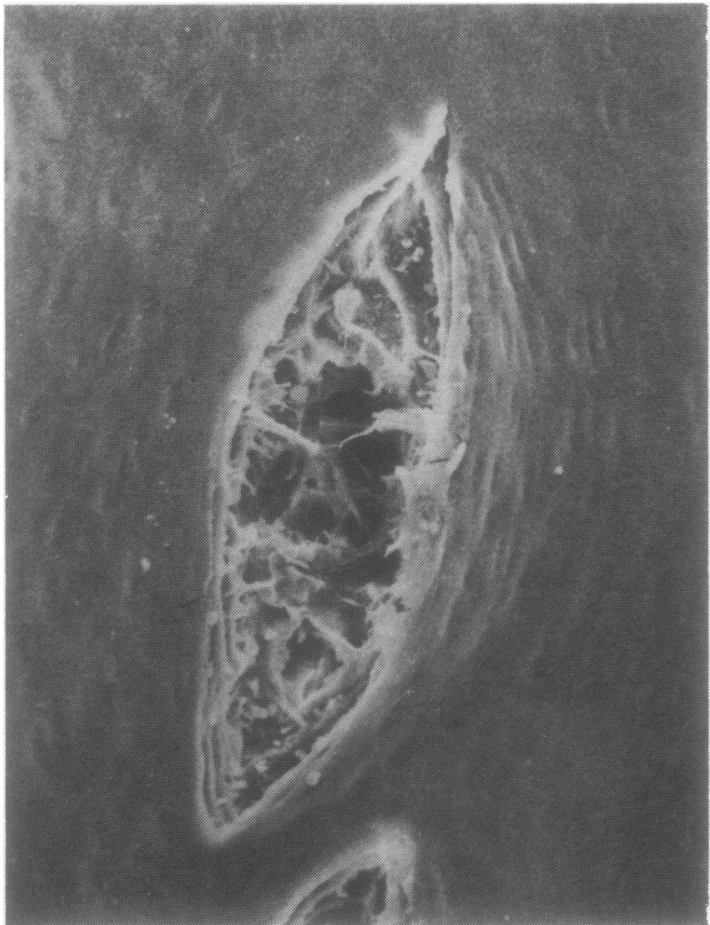

Fig. 2A Unidirectional layered split in non-digested cartilage. (Scanning electron micrograph, $\times 244$ ).

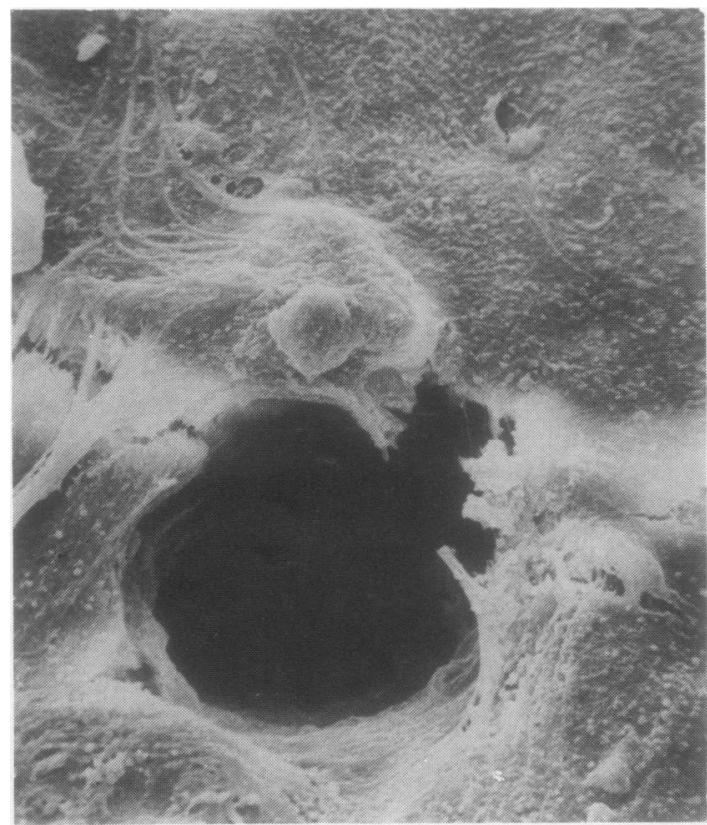

Fig. 2B Round pin-prick after 60 min digestion. (Scanning electron micrograph, $\times 520$ ).

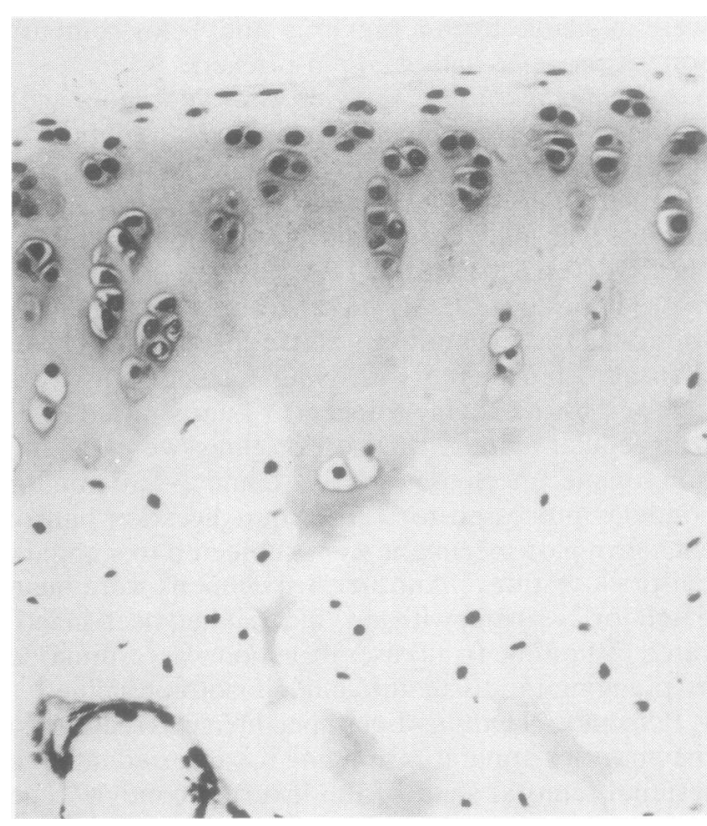

Fig. 3A Unstained band of cartilage at the surface in region 3 after 2 min digestion with papain. (Toluidine blue, $\times 212$ ).

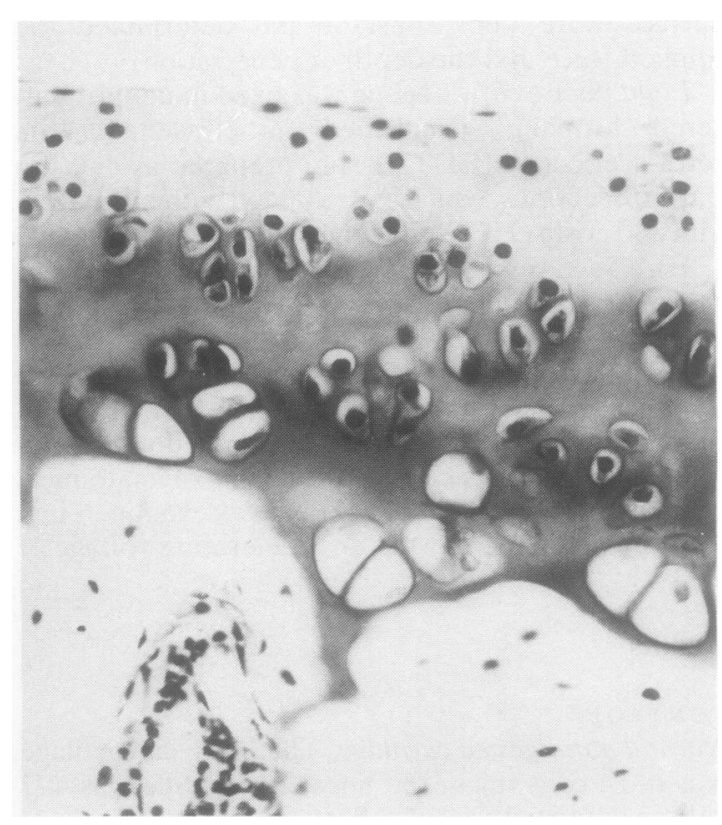

Fig. 3B After 10 min digestion the unstained layer in region 3 has increased. (Toluidine blue, $\times 212$ ). 
band increased with increasing digestion times (Figs. $3 \mathrm{~A}$ and $\mathrm{B}$ ): by $15 \mathrm{~min}$ it had reached $70-85 \%$ of the uncalcified cartilage in region 3 and $100 \%$ in region 1. The first SEM changes were seen after $15 \mathrm{~min}$ digestion, when coarse fibre bundles appeared at the articular margins. These bundles had a parallel, latero-medial arrangement except at the extreme edge of region 1 , where they were organised proximo-distally. Fibres, visible in the central articulating areas conformed to a characteristic pattern; in regions 1 and 2 the fibres were arranged parallel to the longitudinal axis of the specimen, those in regions 3 and 4 were transverse to the axis. An increased number of 3ry features, particularly between the fibre bundles, accompanied these early changes. Splits were now reduced in length.

Cartilage degraded for 20-30 minutes. By $20 \mathrm{~min}$ the unstained band had extended into the calcified layer and by $30 \mathrm{~min}$ some areas of cartilage were totally devoid of stain. However, a superficial zone of flattened cells was still retained. Three forms of 3ry structure were seen: (1) an elevation divided into 2 concentric areas (Fig. 4); (2) a dense central area in a loose, fibrous network (Fig. 5); and (3) a distorted body surrounded by a loose mesh of fibres. The latter was restricted to the articular margins. Away from the 3ry features fibres conformed to the characteristic pattern. Associated with these surface appearances were non-layered, elliptical splits.

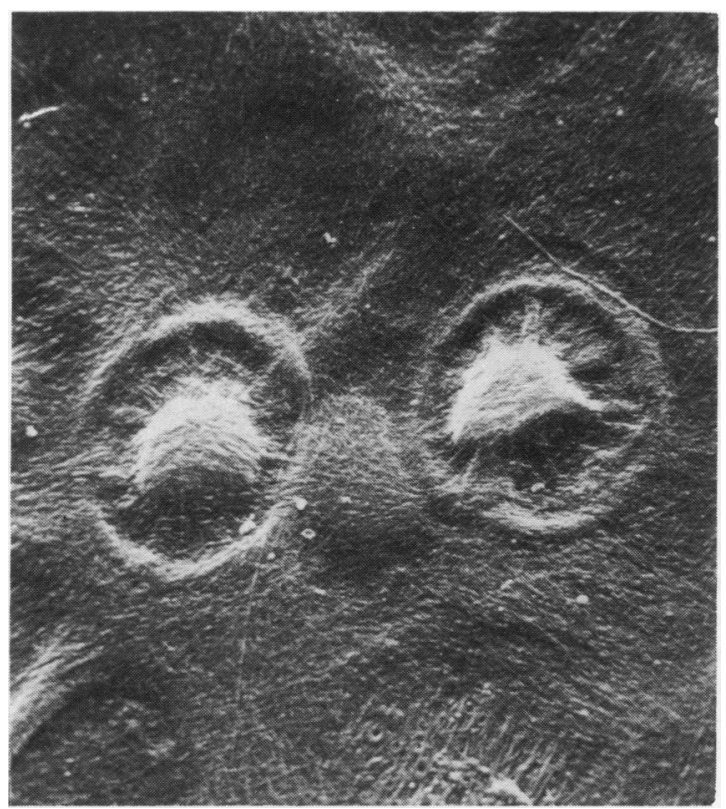

Fig. 4 3ry features after 30 min digestion. (Scanning electron micrograph, $\times 1830$ ).

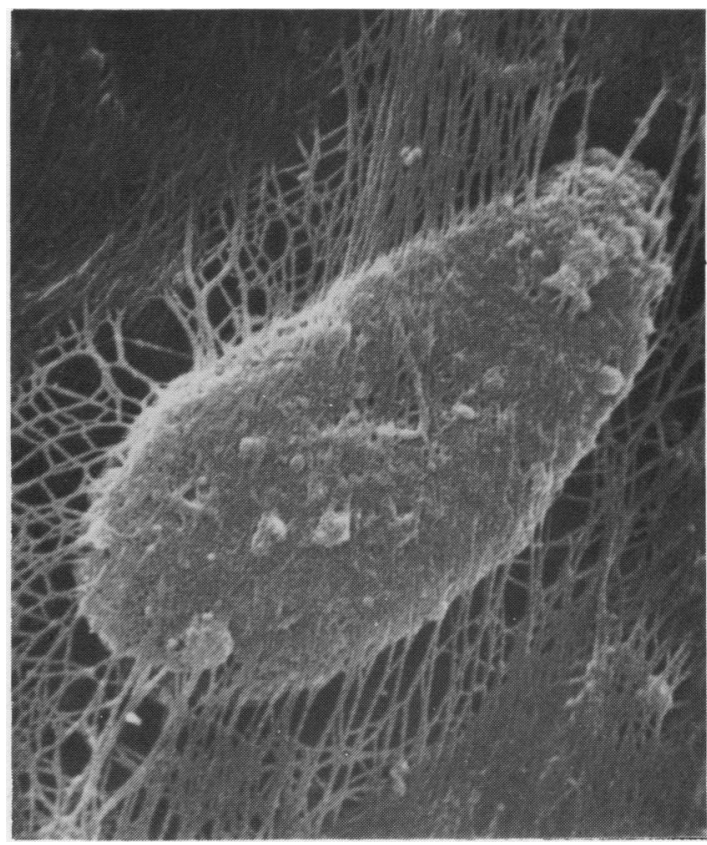

Fig. 5 Suspended $3 r y$ structure after 30 min treatment. (Scanning electron micrograph, $\times 4815$ ).

Cartilage degraded for 60-240 minutes. Enzyme treatment for $60 \mathrm{~min}$ resulted in a reduction in cartilage thickness with a scalloped surface contour in LM section as round lacunae of the deeper zones, III and IV, became exposed. These lacunae now appeared as sharply defined depressions by SEM (Fig. 6). The periarticular fibrous tissue and synovia were completely digested, exposing blood vessel canals of the underlying bone. Round holes with short radiating cracks and reduced secondary cracking resulted from pricking this thinned cartilage (Fig. 2B).

Following $240 \mathrm{~min}$ digestion the calcified cartilage was lost: bone was exposed with trabeculae visible particularly around the condylar groove (Fig. 7). The surface was covered by cracks. Shallow indentations with radiating cracks were produced by pricking exposed bone.

\section{Discussion}

The present results show that enzyme degradation of rat femoral condylar cartilage by the thiol proteinase, papain, causes progressive loss of the metachromatic matrix and topographical changes that lead to complete cartilage loss. The results confirm by direct testing that these changes accompany deterioration in mechanical integrity.

Papain selectively degrades cartilage proteoglycan 


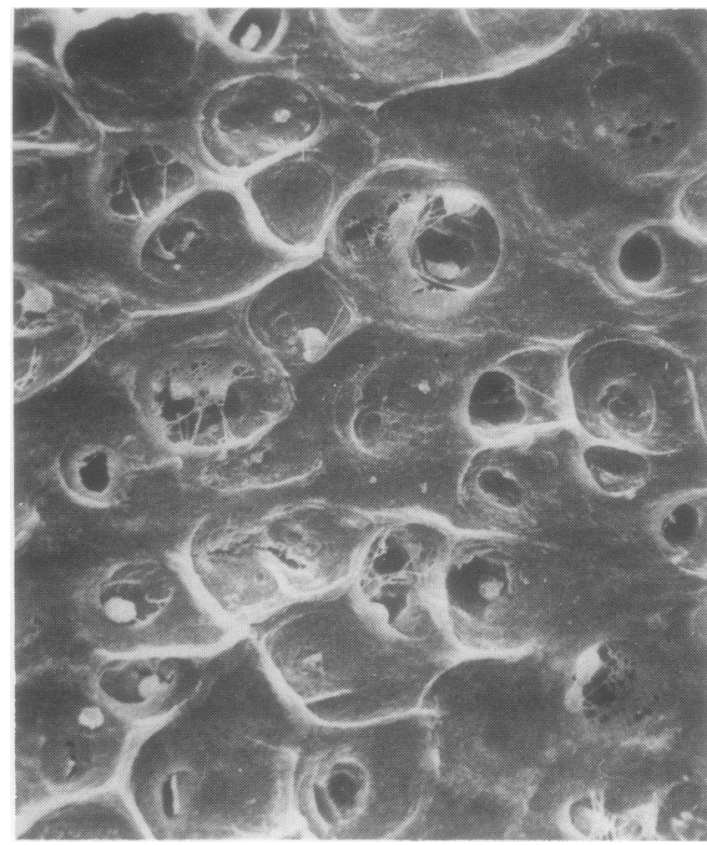

Fig. 6 After 60 min digestion sharply defined depressions with a central hole. (Scanning electron micrograph, $\times 425$ ).

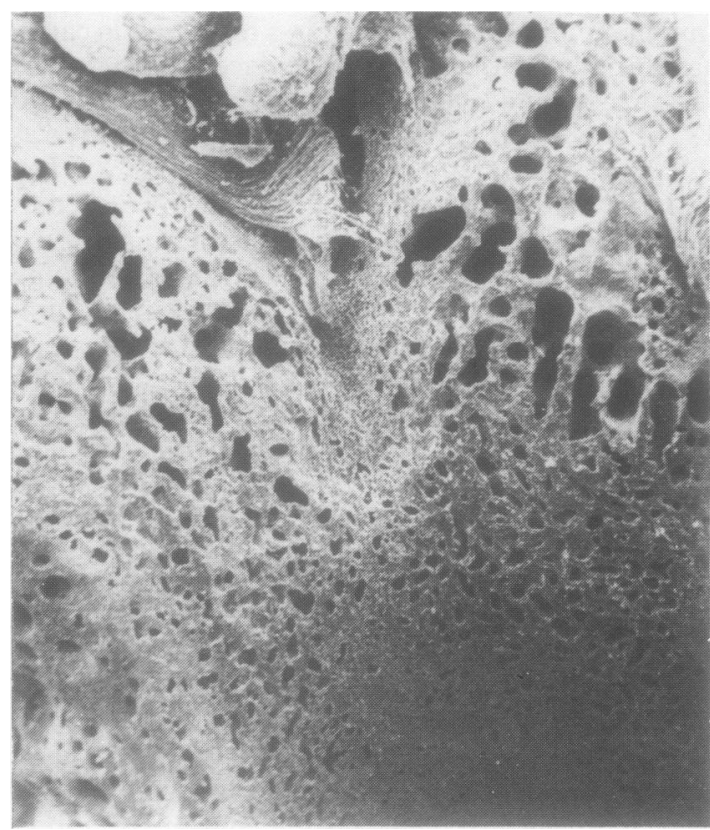

Fig. 7 Exposed bone in region 2, the condylar groove, after 240 min digestion. (Scanning electron micrograph, $\times 19$ ).
(PG), ${ }^{17} 1821$ but collagen is highly resistant to protease digestion. ${ }^{22}$ The changes reported in this paper are due to removal of PG from the entire condylar surface. This initially results in a loss of TB staining from the articular surface layers like that associated with regressive changes in human cartilage. ${ }^{23}$ Poole $^{19}$ has shown that complete loss of TB staining in fresh tissue occurs when hexuronic acid falls below $42 \%$ of the normal cartilage content. Thus after papain digestion at least $58 \%$ of the PG has been disrupted and lost from the unstained areas.

The digestion of cartilage by papain for less than 15 min results in a significant reduction in the capacity of the cartilage to stain with alcoholic TB. Within this period as much as $75 \%$ of the cartilage thickness may lose some PG. It is therefore of the greatest interest that, after this brief period of digestion, the morphology of the cartilage surface, assessed by conventional SEM, should not appear to be changed. It is also significant that at this time a normal shape and pattern of splits is retained after pin-prick testing.

One explanation for this differential loss of PG, with retention of SEM morphology and split patterns, is likely to be the decision to study by conventional SEM preparative techniques material that had been fixed and dehydrated. These methods are not capable of resolving PG molecules and are also notoriously liable to result in the loss of some PG. Collagen fibres are resistant to these punitive techniques and remain apparently intact. If it were possible to adopt preparative procedures, such as very rapid freezing, that conserve water, avoid fixation, and protect PG, and combine these with ultrastructural examination at low temperature, early recognition of the PG loss indicated by TB staining of cartilage sections would be probable. A further investigation is clearly most desirable. It is therefore planned to examine digested articular cartilage by the technique of single-stage, low-temperature replication. This method allows the precision and resolution of TEM to be applied to the study of fully hydrated, natural biological surfaces. Low temperature replication has been used successfully to investigate the surface detail of normal articular surfaces. ${ }^{24}$

As digestion progresses, more PG is lost and collagen fibres and marginal fibre bundles are unmasked. The increase in the number and prominence of 3ry features after preliminary papain digestion suggests that the areas immediately surrounding these structures have a relatively high PG to collagen ratio when compared with the rest of the surface. Removal of PG causes collapse of matrix to form a moat around a central elevation; this is succeeded by exposure of a loose network of fibres as more PG is lost. Tertiary features, present in zone I cartilage, are thought to represent underlying super- 
ficial chondrocytes. They are covered by, and have an intimate association with, the dense mat of collagen fibres that run parallel to the articulating surface. ${ }^{7}{ }^{10}$ Once the midzone is exposed, depressions are seen as cells are lost, implying that collagen fibres are discretely arranged around the midzone chondrocytes.

After 60 min digestion PG loss is accompanied by breakdown and loss of the remaining cartilage components, comprising mainly collagen. There is now a reduction in the overall thickness of the cartilage. Papain may release or activate endogenous collagenases. However, it is probable that removal of the PG packing causes mechanical loosening and, eventually, rupture of the fibre network. The brittle nature of the material seen after 240 min treatment is illustrated by the frequent occurrence of cracks and the presence of lacunae similar to those found in compact bone. ${ }^{25}$

In mechanical terms articular cartilage can be considered as a hydrated gel of PG molecules restrained and reinforced by a collagen fibre network. Papain treatment of this composite preferentially digests the compression-resisting PG matrix. Splits caused in normal cartilage conform with the orientation of anisotropic surface collagen fibres. ${ }^{12}$ The layered and tapered shape of each split is due in part to (1) change in collagen fibre orientation with depth, (2) the compression resistance of $P G$, and (3) the pattern of energy dissipation as new fracture surfaces are created. After brief papain digestion the collagen fibre arrangement appears little affected, though the resistance to compression of the residual PG is already much reduced. This relatively early depletion of $P G$ is one reason why splits appear to reveal no layering and are apparently fractured uniformly throughout their depth. The shortening of the split, the decrease in secondary damage beneath the surface, and the production of round holes subsequently, reflect a reduction in the anisotropy of the tissues, a consequence of exposure of the random fibre network of midzone cartilage. The shallow indentations produced in calcified cartilage and bone by pin-pricking are caused by an increased hardness of the exposed tissue.

Intra-articular injection of papain in vivo ${ }^{15} 212627$ results in severe fibrillation and full thickness cartilage loss. The absence of cleft formation in the present study implies that joint movement is necessary to cause fibrillation by exacerbating the effects of digestion. With or without articulation the end result of papain digestion is full-thickness cartilage loss. The present results support the hypothesis that early loss of PG, such as may occur in osteoarthrosis, ${ }^{23}$ can lead to the weakening and breakdown of all cartilage components, eventually causing cartilage thinning. In a further series of experiments these views will be tested by low-temperature $\mathrm{SEM}^{28}$ and by single-stage replication of frozen hydrated cartilage blocks. ${ }^{24}$

During the course of this study the Department of Histopathology was in receipt of grants from the Arthritis and Rheumatism Council for Research and the Medical Research Council.

\section{References}

1 Bullough P, Goodfellow J. The significance of the fine structure of articular cartilage. J Bone Joint Surg 1968; 50B: 852-7.

2 Gardner D L. The influence of microscopic technology on knowledge of cartilage surface structure. Ann Rheum Dis 1972; 31: $235-58$.

3 Ortmann R. Use of polarized light for quantitive determination of the adjustment of tangential fibers in articular cartilage. Anat Embryol (Berl) 1975; 148: 109-20.

4 Longmore R B, Gardner D L. Development with age of human articular cartilage surface structure. A survey by interference microscopy of the lateral femoral condyle. Ann Rheum Dis 1975; 34: 26-37.

5 McCall J G. Scanning electron microscopy of articular surfaces. Lancet 1968; ii: 1194.

6 Gardner D L, Woodward D. Scanning electron microscopy of articular surfaces. Lancet 1968; ii: 1246.

7 Clarke I C. Surface characteristics of human articular cartilage: a scanning electron microscope study.J Anat 1971; 108: 23-30.

8 Ghadially F N, Ghadially J A, Oryschak A F, Yong N K Experimental production of ridges on rabbit articular cartilage: a scanning electron microscope study.J Anat 1976;121: 119-32.

9 Speer D P, Dahners L. The collagenous architecture of articular cartilage. Correlation of scanning electron microscopy and polarized light microscopy observations. Clin Orthop 1979; 139: 267-75.

10 Weiss C. Normal and osteoarthritic articular cartilage. Orthop Clin North Am 1979; 10: 175-89.

11 Gardner D L, O'Connor P, Oates K. Low temperature scanning electron microscopy of dog and guinea-pig hyaline articular cartilage.J Anat 1981; 132: 267-82.

12 O'Connor P, Bland C, Bjelle A, Gardner D L. Production of split patterns of the articular cartilage surfaces of rats.J Pathol 1980; 130: $15-21$.

13 O'Connor P, Bland C, Gardner D L. Fine structure of artificial splits in femoral condylar cartilage of the rat: a scanning electron microscopic study.J Pathol 1980; 132: 169-79.

14 Thomas L. Reversible collapse of rabbit ears by intravenous papain and prevention of recovery by cortisone. J Exp Med 1956; 104: 245-52.

15 Murray D G. Experimentally induced arthritis using intraarticular papain. Arthritis Rheum 1964; 7: 211-9.

16 Minns R J, Steven F S. The collagen fibril organization in human articular cartilage. J Anat 1977; 123: 437-57.

17 Zambrano N Z, Montes G S, Shigihara K M, Sanchez E M, Junqueira L C U. Collagen arrangement of cartilages. Acta Anat (Basel) 1982; 113: 26-38.

18 Shepard N, Mitchell N. The localization of articular cartilage proteoglycan by electron microscopy. Anat $\operatorname{Rec} 1977$; 187: 463-76.

19 Poole A R. The relationship between toluidine blue staining and hexuronic acid content of cartilage matrix. Histochem J 1970; 2: 425-30.

20 Cameron C H S, Gardner D L, Longmore R B. The preparation of human articular cartilage for scanning electron microscopy. $J$ Microsc 1976; 108: 1-12.

21 Inoue $S$, Glimcher $M J$. The reaction of cartilage and osteophyte formation after the intraarticular injection of papain. J Jpn Orthop Assoc 1982; 56: 415-30. 
22 Stockwell R A. Biology of cartilage cells. Cambridge: Cambridge University Press, 1979.

23 Meachim G, Ghadially F N, Collins D H. Regressive changes in the superficial layer of human articular cartilage. Ann Rheum Dis 1965; 24: 23-30.

24 Gardner D L, O'Connor P, Middleton J F S, Oates K, Orford C R. An investigation by transmission electron microscopy of freeze replicas of dog articular cartilage surfaces: the fibre-rich surface structure. J Anat 1983; 137: 573-82.

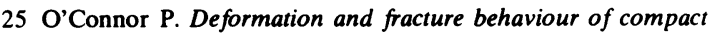
bone. $\mathrm{PhD}$ thesis. University of London, 1976.

26 Bentley G. Papain-induced degenerative arthritis of the hip in rabbits. J Bone Joint Surg 1971; 53B: 324-37.

27 Havdrup T, Telhag H. Papain-induced changes in the knee joints of adult rabbits. Acta Orthop Scand 1977; 48: 143-9.

28 Gardner D L, O’Connor P, Oates K. Low temperature scanning electron microscopy of dog and guinea-pig hyaline articular cartilage. J Anat 1981; 132: 267-82.

\section{Book review}

Bone and Joint Disease in the Elderly. Edited by $\mathrm{V}$. Wright. Pp. 305. $£ 20 \cdot 00$. Churchill Livingstone: Edinburgh. 1983.

In his introduction to this book Bernard Isaacs, professor of geriatric medicine in Birmingham, describes the series of which it forms a part. He writes: 'A series of volumes on Medicine in Old Age is necessary and timely. It is not so very long since teaching hospitals in the United Kingdom did not admit patients over the age of $65 \ldots$. . Now medicine has changed radically and the majority of physicians deal extensively with elderly ill old patients... This new series aims to give doctors working with elderly patients a concise but comprehensive guide to this new knowledge.' Each volume of the series will deal with one system of the body or a closely related group of problems, and this volume covers bone and joint disease.

The first chapter gives an excellent epidemiological appraisal of the subject. There follow chapters on the two giants, osteoarthrosis and rheumatoid arthritis, and then three chapters on the rarer conditions of polymyalgia rheumatica, temporal arteritis, crystal deposition diseases, and the other connective tissue diseases. At this stage a non-specialist reader may feel that he is beginning to be lost in the small print, a feeling likely to be exacerbated by the next chapter, which is a detailed analysis of the associations between rheumatic diseases and cancer. After this osteomalacia and osteoporosis are covered, and then there are two chapters on drugs in rheumatic diseases. The last four chapters of the book cover mobility, physiotherapy, aids and appliances, and orthopaedic surgery.

I think the editor has done well with his selection of titles but not always so well in editing his contributors' writings. The meaning of some sentences is not clear. Misprints are relatively frequent and legends on graphs and tables are sometimes difficult to understand. Named diseases are well covered in the index, as are drugs, but if one starts with a symptom or abnormality the index is less helpful. For example neither 'calcinosis' nor 'nodules' are indexed.

Despite these criticisms I would, on the whole, recommend this book and feel that these small volumes, which can be fairly rapidly produced and are therefore up to date, are an effective way of spreading expertise in the management of the older patient.

C. REISNER 